
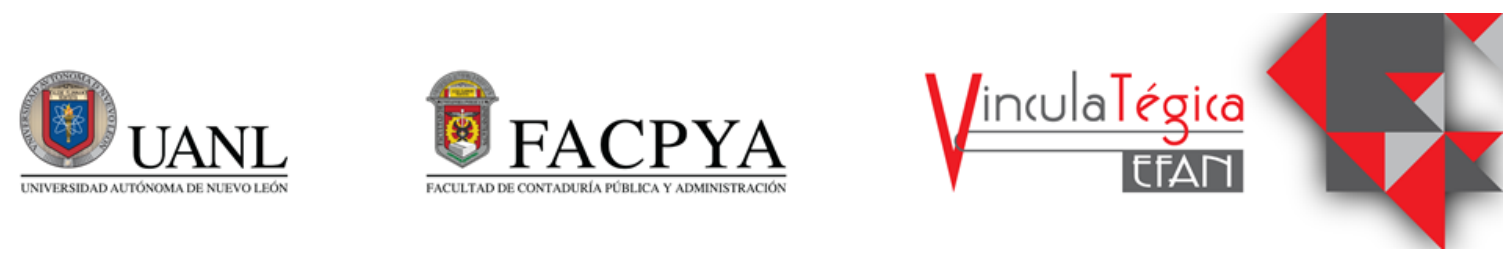

\title{
Turismo y entorno empresarial en los Pueblos Mágicos de Coahuila
}

\author{
César Barboza Lara ${ }^{1}$, Leonor Gutiérrez González ${ }^{2}$ y Horacio Salas Torres ${ }^{3}$ \\ ${ }^{1}$ Universidad Autónoma de Coahuila, Facultad de Contaduría y Administración, \\ cesar.barboza@uadec.edu.mx, Carretera 57,km.4.5 S/N Col. Universidad, (+52) 8661304555 \\ ${ }^{2}$ Universidad Autónoma de Coahuila, Facultad de Contaduría y Administración, \\ leonorgutierrez@uadec.edu.mx, Carretera 57, km.4.5 S/N Col. Universidad, (+52) 8661502703 \\ ${ }^{3}$ Universidad Autónoma de Coahuila, Facultad de Contaduría y Administración, \\ luissalastorres@uadec.edu.mx, Carretera 57,km.4.5 S/N Col.Universidad, (+52) 8661366280
}

Información del artículo revisado por pares

Fecha de aceptación: junio-2021

Fecha de publicación en línea: diciembre-2021

DOI: https://doi.org/10.29105/vtga7.1-98

\begin{abstract}
Resumen
Considerando que la actividad turística genera crecimiento económico y bienestar, en este artículo se estudia la dinámica empresarial de los pueblos mágicos del estado de Coahuila. El objetivo general de este artículo consiste en analizar el número de unidades económicas en cada pueblo mágico por sector de actividad a partir de su nombramiento. Se parte de la hipótesis de que, debido a su nombramiento como pueblo mágico, el turismo contribuyó a impulsar los sectores de actividad económica vinculadas al turismo en cada pueblo mágico, tales como hoteles, restaurantes, servicios de esparcimiento, entre otros. Se utilizan los Censos Económicos del INEGI del 2003, 2008, que muestran para cada pueblo mágico y para cada año, el total de unidades económicas por sector de actividad. Específicamente se analizan dos sectores, el 71 y 72: servicios de esparcimiento culturales, deportivos y recreativos; y los servicios de alojamiento temporal y de preparación de alimentos y bebidas. Los resultados permiten agrupar a los pueblos mágicos en dos clasificaciones: Arteaga, Parras y Cuatro Ciénegas con un efecto importante en el crecimiento de unidades económicas dedicadas al turismo; y Candela, Guerrero y Viesca, que son lugares más pequeños en donde aún no se observa un impacto significativo en el número de unidades económicas.
\end{abstract}

Palabras clave: Pueblos Mágicos, Turismo, Unidades Económicas.

\begin{abstract}
Considering that tourist activity generates economic growth and well-being, this article studies the business dynamics of Pueblos Mágicos of the state of Coahuila. The purpose of this article is to analyze the number of economic units in each town by activity sector. It is based on the hypothesis that, due to its appointment as a Pueblo Mágico, tourism helped to promote the sectors of economic activity linked to tourism in each town, such as hotels, restaurants, recreational services, among others. The INEGI Economic Censuses of 2003, 2008 are used, which show for each town and for each year, the total economic units by activity sector. Two sectors are specifically analyzed, 71 and 72 : cultural, sports and recreational leisure services; and temporary accommodation and food and beverage preparation services. The results allow the towns to be grouped into two classifications: Arteaga, Parras and Cuatro Cienegas with an important effect on the growth of economic units dedicated to tourism; and Candela, Guerrero and Viesca, which are smaller places where a significant impact on the number of economic units has not yet been observed.
\end{abstract}

Keywords: Tourism, Magical Towns, Economic Units JEL: L80, L83, L88. 


\section{INTRODUCCIÓN}

Los municipios crecen y se desarrollan a partir del análisis de una serie de factores de tipo económico, político o social. En general, una de las formas de estudiar el desarrollo local de un territorio se lleva a cabo mediante la identificación del número de empresas nuevas que se instalan en las ciudades. El comportamiento de la variable de la creación de empresas es importante porque tiene repercusiones en otros indicadores, como la creación de empleo y bienestar para los habitantes. Bajo esta óptica, el objetivo principal de este trabajo consiste en analizar los principales cambios en el número de unidades económicas en los pueblos mágicos del estado de Coahuila, a partir de la información proporcionada por los Censos Económicos del Instituto Nacional de Estadística y Geografía (INEGI) del 2003, 2008, 2013 y 2018. Se persigue este objetivo bajo el argumento de que el turismo contribuye a mejorar las condiciones económicas de un determinado lugar.

El Programa Pueblos Mágicos (PPM) es una estrategia de la Secretaría de Turismo de México que inicia en el año 2001, con el propósito de impulsar el desarrollo de pequeñas localidades, lo cual se realiza "a través de la diversificación de mercados y de la competitividad, además, en el marco municipal, por las características específicas del programa que enfatiza la creación de negocios y empleos en el espacio local en pequeñas localidades" (Fernández, 2016).

En general, se ha identificado a esta política como exitosa, ya que ha contribuido al mejoramiento de algunos indicadores económicos en las localidades en donde se ha implementado. Este efecto positivo se observa en mejoras "en infraestructura y servicios, la generación de empleos y creación de pequeñas y medianas empresas; además ha logrado, en algunos casos, disminuir la migración de la población, debido a la reactivación de actividades productivas" (Equihua et al., 2015).

Actualmente, en Coahuila existen siete pueblos mágicos, los cuales son: Arteaga, Candela, Cuatro Ciénegas, Guerrero,
Muzquiz, Parras de la Fuente y Viesca. Esto implica que el Estado ha impulsado esta política como opción de desarrollo local en los destinos que cuentan con los requisitos para ser Pueblo Mágico.

\section{MARCO TEÓRICO}

El argumento principal acerca de la implementación del Programa Pueblos Mágicos es que el turismo funcione como un detonador de la economía de cada localidad seleccionada, y que sirva como "herramienta de desarrollo sustentable y que funcione como apoyo en la gestión municipal, por ejemplo, en la promoción turística, la imagen urbana y la ampliación de servicios" (Hofmann, 2015).

Según la Secretaría de Turismo (2020), un pueblo mágico se define como: "una localidad que tiene atributos únicos, simbólicos, historias auténticas, hechos trascendentes, cotidianidad, que significa una gran oportunidad para el aprovechamiento turístico atendiendo a las motivaciones y necesidades de los viajeros". El programa dio inicio en el 2001 con la denominación de Huasca de Ocampo, Hidalgo y Real de Catorce, San Luis Potosí (Lopez et al., 2015). A 20 años de operación, actualmente se cuentan con 132 Pueblos Mágicos en el país. El nacimiento de esa estrategia se dio "en respuesta a que hasta ese momento no existía política alguna para la promoción de los pueblos o pequeñas ciudades cuyas costumbres, tradiciones, folklore, arquitectura y personajes fuesen atractivas para el turismo cultural, así como también por la carencia de programas de desarrollo local" (Equihua et al., 2015). Esto implicó dar un cambio importante en la política de turismo en México, al pasar de una promoción de los centros de playa, a un turismo más cultural, lo que sin duda beneficiaría a comunidades rezagadas o que carecían de inversión pública.

Uno de los aspectos más importantes en la aplicación del PPM, es que es necesaria la participación comunitaria para alcanzar el éxito. De esta manera, "se pide a los pobladores involucrarse en los talleres de inducción del Programa y de Planeación y Gestión de Turismo Cultural, sobre todo a quienes podrían ofrecer la gastronomía típica 
y el alojamiento; sin embargo, son pocas las acciones encaminadas a la creación de actividades de recreación, esparcimiento y diversión" (Velarde et al., 2009), lo que sin duda representa una fuerte oportunidad de negocio para los pueblos de Coahuila.

En suma, el programa fue creado con el objetivo de desarrollar a las localidades beneficiadas mediante una especialización económica, "esperando que el crecimiento de los servicios relativos al turismo tenga efectos multiplicadores en otras áreas" (García, 2014). Estos efectos pueden ser visibles en la generación de empresas y negocios para los habitantes de cada municipio.

Es decir, si uno de los objetivos más importantes del PPM es el que "las comunidades receptoras de las localidades participantes aprovechen y se beneficien del turismo como actividad redituable y sean una opción de negocio, de trabajo y de forma de vida" (Rosas et al., 2017), cabe hacerse la pregunta de ¿en qué medida esto ha sido cierto para los pueblos mágicos de Coahuila?

En relación a los efectos del programa, a dos décadas del inicio de operaciones de esta política, se han realizado algunas investigaciones orientadas a estudiar el impacto de su aplicación en algunos pueblos mágicos, ya que pertenecer al programa "significa obtener recursos económicos para mejora de la localidad, además de mayor promoción y, por lo tanto, mayor afluencia turística" (Equihua et al., 2015).

$\mathrm{Al}$ respecto, se destaca el estudio realizado por Fernández (2016) que afirma que, en relación a la generación de empleo, "ha habido un crecimiento de $90.8 \%$ en cuanto al trabajo en el sector turístico, $5.1 \%$ sobre la tasa de crecimiento de empleo en general. Referente al primer dato y en concreto se menciona que pasó de 1337 personas ocupadas en 2005 a 2 551 en 2010".

Otra investigación, desarrollada por Hofmann (2015), señala el impacto positivo en algunos pueblos mágicos en el país. En el caso del estado de Puebla, que actualmente tiene 9 localidades inscritas en el programa, se ha duplicado el número de visitantes y la derrama económica ha aumentado en $130 \%$. En Real de Catorce se incrementó en $1300 \%$ el número de visitantes, $670 \%$ la derrama económica y en $400 \%$ la oferta de hospedaje. Por su parte, también estudió el caso de Tequila, en Jalisco, que pasó de recibir 18,000 turistas en 2003 a 165,000 en 2013 (Hofmann, 2015).

Otros pueblos mágicos que también han sido objeto de estudio fueron Huasca, Cholula, Malinalco y Tlayacapan, en donde "se ha comprobado que para el sector turístico restauranteros y hoteleros principalmente- ha habido beneficios, así y como se han generado algunos empleos para cubrir sus necesidades, por lo que las y los trabajadores parecen contentos al no tener que migrar" (Fernández, 2016).

Las investigaciones realizadas hasta este momento se han llevado a cabo en Pueblos Mágicos específicos, ubicados en estados con fuerte vocación turística, como los mencionados previamente, pero han sido escasos los trabajos enfocados en estudiar el caso de los pueblos mágicos de Coahuila, particularmente el efecto a nivel empresarial y de negocios que tiene cada localidad al ser inscrita al programa.

Como se ha insistido, el PPM representa una estrategia exitosa para la atracción de turistas, pero a nivel local, se desconoce si esto también contribuye a generar más empresas, empleo, y negocios orientados a satisfacer las necesidades del flujo turístico, y si estas empresas han sido realmente exitosas, ya que el acuerdo general entre los estudiosos del tema es que los "Pueblos Mágicos representan una verdadera alternativa de progreso en el país debido a que permite el impulso de la actividad turística, con ello el aumento del número de visitantes y la inversión pública, lo que supone mayores posibilidades de desarrollo para las localidades con el nombramiento" (Equihua et al., 2015).

En Coahuila, actualmente existen siete pueblos mágicos. En general se trata de localidades pequeñas con atractivos específicos que buscan atraer visitantes para impulsar el desarrollo de las regiones en donde se encuentra. El estado de Coahuila se integra de cinco regiones socioeconómicas (Aguirre et al., 2018), y en cada una de ellas se encuentra, al menos, un pueblo mágico. En la región sureste se ubica Parras de la Fuente, que fue el 
primer municipio que se incorporó al PPM en el estado y en el norte de México, en el año 2004, además de Arteaga, que se adhirió en 2012. En la región Laguna se cuenta con un pueblo mágico desde 2012, Viesca. En la región centro-desierto se ubica Candela, que desde el 2015 es pueblo mágico, y Cuatro Ciénegas, a partir de 2012. En la región Norte o Fronteriza se localiza Guerrero, que desde el 2015 tiene el nombramiento. Finalmente, en la región carbonífera se encuentra el municipio coahuilense que más recientemente obtuvo la distinción de ser Pueblo Mágico, Melchor Muzquiz, en el 2018.

Estas siete localidades conforman la oferta de Pueblos Mágicos coahuilenses, caracterizados por ser pequeñas localidades, con poca población, excepto Muzquiz, que cuenta co un total de 71627 habitantes (INEGI, 2021). Le sigue en tamaño de población Parras con 44 472; Arteaga, 29578 personas; Viesca, 20305 y Cuatro Ciénegas, con 12715 habitantes. Los pueblos mágicos más pequeños de Coahuila son Guerrero y Candela, con apenas 1643 habitantes.

Ante este panorama, en la medida en que estos lugares representan "localidades que requieren orientarse para mejorar y optimizar el aprovechamiento racional de sus recursos y atractivos naturales y culturales, fortalecer su infraestructura, la calidad de los servicios, la innovación y desarrollo de sus productos turísticos, el marketing y la tecnificación" (Shaadi et al., 2017), se podría esperar un efecto diferenciado considerando dos aspectos: el tamaño de su población, ya que es muy heterogénea; y la oferta de atractivos turísticos para los visitantes y la cantidad de necesidades por satisfacer, como hoteles, restaurantes, cafeterías, tours por el municipio, renta de automóviles u otro equipo de movilidad, museos entre muchas otras oportunidades de negocio que serán estudiados desde un punto de vista de la demografía empresarial, que será explicada en la siguiente sección.

\section{MÉTODO}

El objetivo general de este artículo consiste en analizar el número de unidades económicas en cada pueblo mágico por sector de actividad a partir de su nombramiento de Pueblo Mágico. La pregunta de investigación que surge al respecto es: ¿Cuál sector de actividad económica mostró los mayores incrementos en cada uno de los pueblos mágicos de Coahuila?, y se parte de la hipótesis de que, debido a su nombramiento, el turismo contribuyó a impulsar los sectores de actividad económica vinculadas al turismo en cada pueblo mágico, tales como hoteles, restaurantes, servicios de esparcimiento, entre otros.

Para verificar la hipótesis planteada, se descargaron y analizaron los datos de los Censos Económicos del INEGI para cuatro periodos: 2003, 2005, 2013 y 2018. En esta fuente de información se presentan, para cada pueblo mágico y para cada año, el total de unidades económicas por sector de actividad: desde agricultura, minería, energía eléctrica, construcción, industria manufacturera, comercio al por menor y al por mayor, hasta la variable de interés, que son los sectores vinculados al turismo. Específicamente se analizan dos sectores, el 71 y 72 : servicios de esparcimiento culturales, deportivos y recreativos; y los servicios de alojamiento temporal y de preparación de alimentos y bebidas.

Estos sectores de interés agrupan dentro de esa categoría una amplia variedad de empresas dedicadas al turismo. Es decir, el sector 71 Servicios de esparcimiento culturales y deportivos, y otros servicios recreativos, incluye a: compañías de teatro, danza, grupos musicales, promotores de espectáculos artísticos, culturales y deportivos, museos, sitios históricos, zoológicos, parques con instalaciones recreativas y casa de juego electrónico, casinos, loterías, entre otros. Por su parte, el sector 72 Servicios de alojamiento temporal y de preparación de alimentos y bebidas, incluye a: hoteles, moteles, campamentos, albergues, pensiones, casas de huéspedes, departamentos y casas amuebladas con servicio de hotelería, restaurantes, centros nocturnos, bares, cantinas, cafeterías, fuentes de sodas, neverías, entre muchos otros negocios orientados a los servicios turísticos.

El enfoque de investigación es cuantitativo de tipo descriptivo, ya que se compararon los datos para cada pueblo mágico a partir del año 
del censo más cercano al nombramiento de Pueblo Mágico y se calculó la tasa de variación entre el número de unidades económicas. Por ejemplo, dado que Cuatro Ciénegas obtuvo el nombramiento de Pueblo Mágico en el 2012, se compararon principalmente los datos de los Censos Económicos del 2013 y del 2018, para ver el cambio en la tasa de variación y la proporción que representan las unidades económicas por sector.

La unidad de análisis son los establecimientos, que el INEGI (2016) define como: la unidad económica que, en una sola ubicación física, asentada en un lugar de manera permanente y delimitada por construcciones o instalaciones fijas, combina acciones y recursos bajo el control de una sola entidad propietaria o controlador, para realizar actividades de producción de bienes, compraventa de mercancías o prestación de servicios. Esta definición no está exenta de deficiencias en su medición, ya que no se incluyen los negocios denominados como ambulantes, o con instalaciones no sujetas al suelo, ni aquellos puestos que son armados $y$ desarmados todos los días. De la misma forma, tampoco se incluyen las casas-habitación en donde se realiza alguna actividad productiva, ni los micronegocios que prestan servicios que se implementa en otro lugar, tales como los pintores, plomeros, etc.

En el aspecto espacial, se analizan 6 de los 7 pueblos mágicos, ya que se excluyó Muzquiz porque su nombramiento fue en el 2018, por lo que no era posible observar una tendencia en el tiempo.

\section{RESULTADOS}

En esta sección se incluyen los principales resultados para cada pueblo mágico por orden alfabético. En primer lugar, el cuadro 1 muestra los principales resultados de Arteaga, que desde el año 2012 tiene el nombramiento de pueblo mágico. El total de unidades económicas pasó de 247 en el 2003 a 424 en el 2018. El periodo intercensal de mayor crecimiento en el número de negocios fue entre 2013 y 2018, precisamente después de haber sido nombrado Pueblo Mágico: entre 2003 y 2008 el número de unidades económicas crecieron apenas $4 \%$, pero entre el 2013 y el 2018, el crecimiento fue del $30 \%$. Esta cifra sin duda habla de un impacto positivo en el entorno económico de Arteaga posterior a su nombramiento como Pueblo Mágico.

Algunos de los principales hallazgos de estos datos son, en primer lugar, que el comercio al por menor bajó de manera importante, ya que en el 2003 representaba casi el 50\% de todas las unidades económicas del municipio, pero para el 2018, la cifra se redujo a $36 \%$, mientras que, en segundo lugar, un sector que creció fue la industria manufacturera (de 25 a 72 en quince años).

Respecto al sector del turismo, se observa un impacto positivo en la creación de nuevas unidades económicas. En el Censo Económico del 2013, un año después del nombramiento como Pueblo Mágico, el sector de alojamiento $\mathrm{y}$ de preparación de alimentos y bebidas representaba el $8.3 \%$ del total de unidades económicas del municipio, pero cuatro años después, dicha proporción se incrementó a $13 \%$.

Este sector económico concentra los negocios enfocados a las diversas actividades de hospedaje, como hoteles, moteles, campamentos, casas de huéspedes, pensiones y cabañas. Este último tipo de negocio se encuentra en constante crecimiento en Arteaga, y de hecho el pueblo es conocido como la "Suiza de México" por los paisajes boscosos y cabañas, por lo que es un sector económico importante para el municipio.

Además, este sector 71 también aglomera los negocios enfocados a la preparación de alimentos y bebidas, entre los que se incluye a todo tipo de restaurantes, centros nocturnos o bares, los cuales son negocios enfocados al turismo.

Contrario a lo que sucede en Arteaga, en el Pueblo Mágico de Candela el impacto del turismo no es tan notorio si se analiza el comportamiento del número de unidades económicas en los periodos intercensales. Sin embargo, es importante destacar que Candela obtuvo el nombramiento de Pueblo Mágico en 2015, tres años después de Arteaga. El cuadro 2 presenta los resultados para este municipio.

Candela, al ser un pequeño municipio de 
menos de 2,000 habitantes, según el último Censo de Población y Vivienda, también se caracteriza por contar con pocas unidades económicas, con 59 en el 2018. Al igual que Arteaga, la actividad económica preponderante es el Comercio al por menor, que representó el 58\% en el 2018, seguido de otros servicios (15\%), industrias manufactureras (10\%) y los servicios de alojamiento temporal y de preparación de alimentos y bebidas con el $8 \%$. Un hallazgo importante sobre este municipio, es que no registra unidades económicas dentro del sector de Servicios de Esparcimiento culturales y deportivos, al menos hasta el 2018, ya que no se habían instalado empresas relacionadas con ese sector, lo cual representa una importante área de oportunidad para el mejoramiento de la infraestructura del Pueblo Mágico de Candela.

El tercer pueblo mágico de Coahuila que se analiza es el de Cuatro Ciénegas, que se ha caracterizado por un mayor nivel de reconocimiento a nivel nacional, e incluso internacional, por sus atractivos naturales y turísticos. Este municipio obtuvo el nombramiento de Pueblo Mágico en el 2012, hace casi 10 años, por lo que se esperaría que fuera más notorio el impacto en la creación de negocios enfocados al sector turismo. En general, el número de unidades económicas se ha mantenido relativamente estable: 395 en el 2003; 413 en el 2008; 417 en el 2013 y 420 en el 2018 (cuadro 3).

Sin embargo, en este Pueblo Mágico sí es especialmente notorio el crecimiento en términos absolutos que tuvo el rubro de Servicios de alojamiento y preparación de alimentos, ya que fue el que más creció en el periodo intercensal en el que se nombró Pueblo Mágico a Cuatro Ciénegas entre 2013 al 2018, cuando se registraron 11 nuevas unidades económicas, tendencia que continúa actualmente con la instalación y apertura de más hoteles, restaurantes, empresas prestadoras de servicios turísticos, entre otras que impactan de manera importante el crecimiento económico del municipio. $\mathrm{Al}$ 2018, cerca de 500 personas trabajaban en los sectores de esparcimiento, alojamiento y preparación de alimentos y bebidas en Cuatro
Ciénegas.

Un cuarto municipio que también es Pueblo Mágico en Coahuila es Guerrero, ubicado al norte del estado, muy cerca de la frontera con Estados Unidos. Este pequeño municipio de apenas 1643 habitantes obtuvo su nombramiento de Pueblo Mágico en el año 2015, por lo que el impacto en términos del crecimiento y dinámica empresarial ha sido lento, lo cual se ve reflejado en el cuadro 4, que presenta las estadísticas de las unidades económicas de Guerrero para cuatro censos económicos.

Como se puede observar en el cuadro 4, el número de unidades económicas son pocas en el pueblo mágico de Guerrero, aunque sí se aprecia un crecimiento: de 15 en el 2003 a 25 en el 2018. El comercio al por menor, como en los demás municipios, continúa siendo la actividad preponderante. Por lo que respecta a la cuestión de los negocios enfocados al turismo, se resaltan dos aspectos: en primer lugar, no se registra actividad empresarial en el rubro de servicios de esparcimiento culturales y deportivos, lo cual representa un área de oportunidad para el municipio para fomentar la inversión en este sector económico, ya que sí cuenta con espacios destinados a la caza que pueden ser aprovechados para crear más negocios de atracción del turista. En segundo lugar, lo que tiene que ver con servicios de alojamiento y preparación de alimentos y bebidas, cabe señalar que en el 2003 no se contaba con unidades económicas de este tipo, cifra que para el siguiente ejercicio censal se incrementó a 6, pero que descendió a 4 en el 2018. Entonces, se observa una dinámica particularmente lenta pero que sí registra una tendencia hacia un mejoramiento de la infraestructura turística.

El quinto municipio de análisis es Parras de la Fuente, que se caracteriza porque fue el primer pueblo mágico del norte de México, ya que obtuvo su nombramiento en el año 2004. Este destino turístico es el más consolidado del estado, ya que tiene una amplia oferta de sitios turísticos, particularmente enfocados a la producción de vino, con grandes extensiones de tierra dedicados al cultivo de la uva, lo que repercute en el entorno empresarial y de 
creación de unidades económicas del pueblo. El cuadro 5 presenta la información de las unidades económicas de este municipio. En los más de 15 años desde su nombramiento, se han triplicado las unidades económicas orientadas a los servicios de esparcimiento culturales y deportivos, y se ha duplicado el número de empresas dedicadas al servicio de alojamiento temporal y de preparación de alimentos y bebidas. De hecho, este rubro es el tercer más importante del municipio con el $13 \%$ de todas las unidades económicas, según el último censo económico del 2018. Esta misma fuente de información señala que, en conjunto, ambos sectores económicos dan empleo a cerca de mil personas en el municipio, cuando hace 18 años esta cantidad era de 350 personas empleadas en ambos sectores. Sin duda el efecto del turismo y del nombramiento de pueblo mágico tuvo un impacto positivo en la generación y permanencia de unidades económicas en Parras.

Finalmente se describe el municipio de Viesca, que es pueblo mágico desde el año 2012. El último Censo Económico del año 2018 señala que existían 145 unidades económicas en Viesca, un crecimiento bajo en comparación con el 2013, cuando había 112 empresas, según se observa en el cuadro 6 , a pesar de que cuenta con una población de 20 mil personas.

Los datos registrados en los censos económicos indican que, en Viesca, Coahuila, las unidades económicas han tenido un comportamiento diferenciado: entre el 2003 y el 2008 se incrementó de 94 a 130 el número de unidades económicas, una tasa de variación del 38\%. Sin embargo, entre 2008 y 2013 dicha tasa mostró un comportamiento negativo del $14 \%$, para luego recuperarse de manera importante después del nombramiento de pueblo mágico, cuando en el 2013 pasó de 112 unidades económicas, a 145 en el 2018, una variación de $29 \%$.

El rubro de los servicios de alojamiento y preparación de alimentos y bebidas se duplicó a partir del nombramiento: 13 en el 2013 y 26 en el 2018, un impacto relevante para el desarrollo económico del pueblo. En ese año, las empresas orientadas a este servicio representaron el $18 \%$ de todas las unidades económicas del municipio, mientras que, el comercio al por menor, como en todos los casos analizados, es la actividad económica preponderante en Viesca, con el $48 \%$ del total de las unidades económicas. En este sentido, en casi 10 años desde que es pueblo mágico, en Viesca se ve un impacto positivo del turismo, a pesar de que no hay registro de las unidades económicas enfocadas a los servicios de esparcimiento, lo cual también representa una importante área de oportunidad en la medida en que se cuenta con atractivos naturales que pueden impulsar la creación de negocios de esparcimiento para el turista.

\section{CONCLUSIONES}

A lo largo de distintas investigaciones en diferentes contextos regionales, ya sea en México o en el exterior, se ha podido comprobar que el turismo es un generador de crecimiento económico medido a partir de la generación de empresas y un mayor sector consolidado de servicios en los diferentes destinos.

Tradicionalmente no se asocia al estado de Coahuila como un destino turístico de alto impacto, puesto que es parte de un país con una gran oferta turística, principalmente enfocada al turismo de playas. De hecho, según el Barómetro del Turismo Mundial de la Organización Mundial del Turismo (2020), México fue el tercer país más visitado en el mundo durante diciembre del 2020. A pesar de que esta situación tuvo que ver con el contexto de pandemia actual, es innegable también que México ha sido uno de los países más visitados del mundo.

Sin embargo, en este artículo, el objetivo principal se enfocó en otro segmento del mercado turístico mexicano: los pueblos mágicos, una política del gobierno federal que nació hace 20 años, y que cada año busca consolidar su permanencia como una opción más para el turista.

El estado de Coahuila no ha escapado a esta realidad. Actualmente es de los estados que más Pueblos Mágicos tiene en todo México, con un total de 7. De esta manera, su oferta se ha ampliado y ha buscado consolidar la permanencia de cada uno de los pueblos 
mágicos con los que cuenta, aumentando la oferta de atractivos turísticos de la mano de los empresarios de cada localidad. De hecho, han sido ellos quienes han ido innovando $y$ adaptándose a las demandas que emanan de los crecientes flujos turísticos, con algunas diferencias.

Precisamente ese fue el objetivo de este artículo: analizar la dinámica empresarial de los negocios en cada pueblo mágico del estado. Al respecto, una de las principales conclusiones es que el hecho de que un lugar sea nombrado como Pueblo Mágico podría tener efectos positivos en la creación de unidades económicas, aunque es necesario considerar el tamaño de cada uno de ellos.

Lo anterior quiere decir que el efecto positivo del nombramiento de Pueblo Mágico se observó particularmente en tres destinos: Parras, Arteaga y Cuatro Ciénegas, que son los más consolidados y de mayor tamaño entre los pueblos mágicos de Coahuila. Por su parte, en lugares como Viesca, Guerrero y Candela, aun no se presenta un incremento significativo en el número de unidades económicas enfocadas al turismo, lo que, más allá de su tamaño, podría deberse a otros factores, como el tiempo que ha transcurrido desde el nombramiento, la inversión que se ha hecho en promoción al turismo, la oferta turística del pueblo, la creación de negocios enfocados a satisfacer necesidades específicas del turista, entre otros aspectos.

En todos los casos, el sector de actividad económica que más ha crecido es el comercio al por menor, que incluye una gran variedad de negocios: comercio de abarrotes, alimentos, bebidas, hielo y tabaco; venta de productos textiles, bisutería, accesorios de vestir y calzado; comercio de artículos para el cuidado a la salud; papelería; enseres domésticos, computadoras, ferretería, entre muchos otros. Es decir, son las empresas que predominan en cada uno de los pueblos mágicos.

Sin embargo, es importante resaltar el papel creciente que están teniendo las unidades económicas enfocadas al turismo, como el hospedaje y la alimentación. Medido como proporción, dicho indicador va del $8 \%$ en Candela, hasta el $18 \%$ de todas las unidades económicas en Viesca. Un dato interesante es que, en Arteaga, Cuatro Ciénegas y Parras, los pueblos mágicos de mayor consolidación, las unidades económicas de este sector representan el $13 \%$ de todos los negocios.

En cuanto a la industria del hospedaje, una tendencia que se observa en los pueblos mágicos es que, en la medida en que consoliden su vocación turística, mayor será su especialización económica enfocada a este rubro, lo que se traduce en mayor cantidad de establecimientos de alojamiento. Más allá del incremento en el número de hoteles, lo cual sí está ocurriendo en Parras, Cuatro Ciénegas y Arteaga, cada vez es más notoria la presencia de opciones alternativas para cumplir con la demanda de hospedaje: esto se traduce en mayores casas registradas en la plataforma Airbnb, que es la aplicación móvil que permite a los turistas alojarse en viviendas locales. Adicionalmente se observa un mayor número de viviendas con opción de renta diaria, semanal o mensual, principalmente en los pueblos mágicos de Parras o Cuatro Ciénegas. Es decir, el panorama del hospedaje en los destinos más consolidados ha cambiado mucho y dista mucho de la escena de hace 5 o 6 años, lo que se refleja en los registros de los Censos Económicos del Inegi y de los mismos registros que llevan las Direcciones de Turismo de cada municipio.

Por otra parte, es importante señalar un área de oportunidad para el turismo en cada pueblo mágico. El sector 71, de servicios de esparcimiento, culturales y deportivos, muestra poca representación en casi todos los pueblos mágicos, lo que significa que es necesario impulsar este tipo de negocios en los pueblos mágicos para atraer más turismo $\mathrm{y}$, por lo tanto, mayores beneficios económicos para la población a través del empleo.

Sin duda, en la medida en que se consoliden los destinos, mayor serán los registros en los periodos censales en que se levante información sobre las unidades económicas en cada Pueblo Mágico, incluido Muzquiz, que fue omitido de este análisis por cuestiones temporales. 
Cuadro 1. Unidades económicas por sector de actividad del Pueblo Mágico de Arteaga, 2003-2018.

\begin{tabular}{|c|c|c|c|c|c|c|c|c|}
\hline \multirow{2}{*}{ Sector Económico } & \multicolumn{2}{|c|}{2003} & \multicolumn{2}{|c|}{2008} & \multicolumn{2}{|c|}{2013} & \multicolumn{2}{c|}{2018} \\
\cline { 2 - 9 } & $\mathrm{UE}$ & $\%$ & $\mathrm{UE}$ & $\%$ & $\mathrm{UE}$ & $\%$ & $\mathrm{UE}$ & $\%$ \\
\hline $\begin{array}{c}\text { Agricultura, explotación } \\
\text { animal, aprovechamiento } \\
\text { forestal, pesca y caza }\end{array}$ & 0 & $0 \%$ & 0 & $0 \%$ & 0 & $0 \%$ & 2 & $0.5 \%$ \\
\hline Minería & 1 & $0.4 \%$ & 2 & $0.8 \%$ & 1 & $0.3 \%$ & 1 & $0.2 \%$ \\
\hline Energía eléctrica, agua y gas & 1 & $0.4 \%$ & 1 & $0.4 \%$ & 1 & $0.3 \%$ & 1 & $0.2 \%$ \\
\hline Construcción & 2 & $0.8 \%$ & 1 & $0.4 \%$ & 0 & $0 \%$ & 0 & $0 \%$ \\
\hline Industria manufacturera & 25 & $10.1 \%$ & 33 & $12.8 \%$ & 48 & $14.7 \%$ & 72 & $17 \%$ \\
\hline Comercio al por mayor & 10 & $4 \%$ & 14 & $5.4 \%$ & 10 & $3.1 \%$ & 20 & $4.7 \%$ \\
\hline Comercio al por menor & 119 & $48.2 \%$ & 122 & $47.5 \%$ & 146 & $44.8 \%$ & 156 & $36.8 \%$ \\
\hline $\begin{array}{c}\text { Transporte, correos y } \\
\text { almacenamiento }\end{array}$ & 8 & $3.2 \%$ & 4 & $1.6 \%$ & 5 & $1.5 \%$ & 13 & $3.1 \%$ \\
\hline $\begin{array}{c}\text { Información en medios } \\
\text { masivos }\end{array}$ & 0 & $0 \%$ & 0 & $0 \%$ & 1 & $0.3 \%$ & 0 & $0 \%$ \\
\hline $\begin{array}{c}\text { Servicios mobiliarios y de } \\
\text { alquiler de bienes muebles }\end{array}$ & 11 & $4.5 \%$ & 10 & $3.9 \%$ & 16 & $4.9 \%$ & 14 & $3.3 \%$ \\
\hline $\begin{array}{c}\text { Servicios profesionales, } \\
\text { científicos y técnicos }\end{array}$ & 2 & $0.8 \%$ & 2 & $0.8 \%$ & 3 & $0.9 \%$ & 6 & $1.4 \%$ \\
\hline $\begin{array}{c}\text { Servicios de apoyo a negocios } \\
\text { y manejo de residuos }\end{array}$ & 1 & $0.4 \%$ & 3 & $1.2 \%$ & 3 & $0.9 \%$ & 6 & $1.4 \%$ \\
\hline Servicios educativos & 2 & $0.8 \%$ & 0 & $0 \%$ & 3 & $0.9 \%$ & 5 & $1.2 \%$ \\
\hline $\begin{array}{c}\text { Servicios de salud y asistencia } \\
\text { social }\end{array}$ & 9 & $3.6 \%$ & 7 & $2.7 \%$ & 11 & $3.4 \%$ & 12 & $2.8 \%$ \\
\hline $\begin{array}{c}\text { Servicios de esparcimiento } \\
\text { culturales y deportivos y otros } \\
\text { servicios recreativos }\end{array}$ & 2 & $0.8 \%$ & 6 & $2.3 \%$ & 3 & $0.9 \%$ & 4 & $0.9 \%$ \\
\hline $\begin{array}{c}\text { Servicios de alojamiento } \\
\text { temporal y de preparación de } \\
\text { alimentos y bebidas }\end{array}$ & 25 & $10.1 \%$ & 28 & $10.9 \%$ & 28 & $8.6 \%$ & 55 & $13 \%$ \\
\hline $\begin{array}{c}\text { Otros servicios excepto } \\
\text { actividades gubernamentales }\end{array}$ & 29 & $11.7 \%$ & 24 & $9.3 \%$ & 47 & $14.4 \%$ & 57 & $13.4 \%$ \\
\hline Total & 247 & $100 \%$ & 257 & $100 \%$ & 326 & $100 \%$ & 424 & $100 \%$ \\
\hline
\end{tabular}

Fuente: Censos Económicos 2003, 2008, 2013 y 2018 del Instituto Nacional de Estadística y Geografí 
Cuadro 2. Unidades económicas por sector de actividad del Pueblo Mágico de Candela, 2003-2018.

\begin{tabular}{|c|c|c|c|c|c|c|c|c|}
\hline \multirow{2}{*}{ Sector Económico } & \multicolumn{2}{|c|}{2003} & \multicolumn{2}{|c|}{2008} & \multicolumn{2}{|c|}{2013} & \multicolumn{2}{|c|}{2018} \\
\hline & UE & $\%$ & UE & $\%$ & $\mathrm{UE}$ & $\%$ & UE & $\%$ \\
\hline Energía eléctrica, agua y gas & 1 & $2 \%$ & 1 & $2 \%$ & 1 & $2 \%$ & 1 & $2 \%$ \\
\hline Construcción & 0 & $0 \%$ & 0 & $0 \%$ & 1 & $2 \%$ & 1 & $2 \%$ \\
\hline Industria manufacturera & 5 & $11 \%$ & 4 & $7 \%$ & 2 & $4 \%$ & 6 & $10 \%$ \\
\hline Comercio al por mayor & 0 & $0 \%$ & 5 & $9 \%$ & 1 & $2 \%$ & 2 & $3 \%$ \\
\hline Comercio al por menor & 32 & $71 \%$ & 33 & $58 \%$ & 35 & $64 \%$ & 34 & $58 \%$ \\
\hline Medios masivos & 1 & $2 \%$ & 0 & $0 \%$ & 0 & $0 \%$ & 0 & $0 \%$ \\
\hline Servicios financieros & 0 & $0 \%$ & 0 & $0 \%$ & 1 & $2 \%$ & 1 & $2 \%$ \\
\hline Servicios mobiliarios & 1 & $2 \%$ & 1 & $2 \%$ & 1 & $2 \%$ & 1 & $2 \%$ \\
\hline $\begin{array}{c}\text { Servicios de apoyo a negocios } \\
\text { y manejo de residuos }\end{array}$ & 0 & $0 \%$ & 2 & $4 \%$ & 1 & $2 \%$ & 0 & $0 \%$ \\
\hline Servicios de salud & 0 & $0 \%$ & 1 & $2 \%$ & 0 & $0 \%$ & 0 & $0 \%$ \\
\hline $\begin{array}{l}\text { Servicios de alojamiento } \\
\text { temporal y de preparación de } \\
\text { alimentos y bebidas }\end{array}$ & 2 & $4 \%$ & 6 & $11 \%$ & 6 & $11 \%$ & 5 & $8 \%$ \\
\hline $\begin{array}{c}\text { Otros servicios excepto } \\
\text { actividades gubernamentales }\end{array}$ & 3 & $7 \%$ & 4 & $7 \%$ & 6 & $11 \%$ & 9 & $15 \%$ \\
\hline Total & 45 & $100 \%$ & 57 & $100 \%$ & 55 & $100 \%$ & 59 & $100 \%$ \\
\hline
\end{tabular}

Fuente: Censos Económicos 2003, 2008, 2013 y 2018 del Instituto Nacional de Estadística y Geografía.

Cuadro 3. Unidades económicas por sector de actividad del Pueblo Mágico de Cuatro Ciénegas, 2003-2018.

\begin{tabular}{|c|c|c|c|c|c|c|c|c|}
\hline \multirow{2}{*}{ Sector Económico } & \multicolumn{2}{|c|}{2003} & \multicolumn{2}{c|}{2008} & \multicolumn{2}{c|}{2013} & \multicolumn{2}{c|}{2018} \\
\cline { 2 - 10 } & UE & $\%$ & UE & $\%$ & UE & $\%$ & UE & $\%$ \\
\hline Agricultura & 0 & $0 \%$ & 0 & $0 \%$ & 2 & $0 \%$ & 2 & $0 \%$ \\
\hline Minería & 5 & $1 \%$ & 4 & $1 \%$ & 6 & $1 \%$ & 4 & $1 \%$ \\
\hline Energía eléctrica, agua y gas & 1 & $0 \%$ & 1 & $0 \%$ & 1 & $0 \%$ & 1 & $0 \%$ \\
\hline Industria manufacturera & 45 & $11 \%$ & 51 & $12 \%$ & 49 & $12 \%$ & 50 & $12 \%$ \\
\hline Comercio al por mayor & 8 & $2 \%$ & 11 & $3 \%$ & 12 & $3 \%$ & 15 & $4 \%$ \\
\hline Comercio al por menor & 207 & $52 \%$ & 201 & $49 \%$ & 199 & $48 \%$ & 189 & $45 \%$ \\
\hline Transporte y correos & 2 & $1 \%$ & 0 & $0 \%$ & 0 & $0 \%$ & 1 & $0 \%$ \\
\hline Información medios masivos & 1 & $0 \%$ & 1 & $0 \%$ & 2 & $0 \%$ & 0 & $0 \%$ \\
\hline $\begin{array}{c}\text { Servicios mobiliarios } \\
\text { Servicios profesionales, } \\
\text { científicos y técnicos }\end{array}$ & 6 & $2 \%$ & 6 & $11 \%$ & 6 & $1 \%$ & 9 & $2 \%$ \\
\hline $\begin{array}{c}\text { Servicios de apoyo a negocios } \\
\text { y manejo de residuos }\end{array}$ & 0 & $0 \%$ & 5 & $1 \%$ & 10 & $2 \%$ & 4 & $1 \%$ \\
\hline $\begin{array}{c}\text { Servicios educativos } \\
\text { Servicios de salud }\end{array}$ & 2 & $1 \%$ & 2 & $0 \%$ & 0 & $0 \%$ & 0 & $0 \%$ \\
\hline $\begin{array}{c}\text { Servicios de esparcimiento } \\
\text { culturales, deportivos y otros } \\
\text { servicios recreativos }\end{array}$ & 4 & $1 \%$ & 6 & $1 \%$ & 5 & $1 \%$ & 6 & $1 \%$ \\
\hline $\begin{array}{c}\text { Servicios de alojamiento } \\
\text { temporal y de preparación de } \\
\text { alimentos y bebidas }\end{array}$ & 34 & $9 \%$ & 48 & $12 \%$ & 44 & $11 \%$ & 55 & $13 \%$ \\
\hline $\begin{array}{c}\text { Otros servicios excepto } \\
\text { actividades gubernamentales }\end{array}$ & 59 & $15 \%$ & 60 & $15 \%$ & 62 & $15 \%$ & 63 & $15 \%$ \\
\hline
\end{tabular}




\begin{tabular}{|l|c|c|c|c|c|c|c|c|}
\hline Total & 395 & $100 \%$ & 413 & $100 \%$ & 417 & $100 \%$ & 420 & $100 \%$ \\
\hline
\end{tabular}

Fuente: Censos Económicos 2003, 2008, 2013 y 2018 del Instituto Nacional de Estadística y Geografía.

Cuadro 4. Unidades económicas por sector de actividad del Pueblo Mágico de Guerrero, 2003-2018.

\begin{tabular}{|c|c|c|c|c|c|c|c|c|}
\hline \multirow[b]{2}{*}{ Sector Económico } & \multicolumn{2}{|c|}{2003} & \multicolumn{2}{|c|}{2008} & \multicolumn{2}{|c|}{2013} & \multicolumn{2}{|c|}{2018} \\
\hline & UE & $\%$ & UE & $\%$ & UE & $\%$ & UE & $\%$ \\
\hline Energía eléctrica, suministro de agua y de gas natural. & 1 & $7 \%$ & 1 & $3 \%$ & 1 & $4 \%$ & 1 & $4 \%$ \\
\hline Industrias manufactureras & 1 & $7 \%$ & 2 & $7 \%$ & 3 & $11 \%$ & 1 & $4 \%$ \\
\hline Comercio al por mayor & 0 & $0 \%$ & 0 & $0 \%$ & 0 & $0 \%$ & 1 & $4 \%$ \\
\hline Comercio al por menor & 10 & $67 \%$ & 16 & $55 \%$ & 15 & $56 \%$ & 15 & $60 \%$ \\
\hline Servicios de alojamiento temporal y de preparación de alimentos y bebidas & 0 & $0 \%$ & 4 & $14 \%$ & 2 & $7 \%$ & 3 & $12 \%$ \\
\hline Otros servicios excepto actividades gubernamentales & 3 & $20 \%$ & 6 & $21 \%$ & 6 & $22 \%$ & 4 & $16 \%$ \\
\hline Total & 15 & & 29 & & 27 & & 25 & \\
\hline
\end{tabular}

Fuente: Censos Económicos 2003, 2008, 2013 y 2018 del Instituto Nacional de Estadística y Geografía.

Cuadro 5. Unidades económicas por sector de actividad del Pueblo Mágico de Parras, 2003-2018.

\begin{tabular}{|c|c|c|c|c|c|c|c|c|}
\hline \multirow[b]{2}{*}{ Sector Económico } & \multicolumn{2}{|c|}{2003} & \multicolumn{2}{|c|}{2008} & \multicolumn{2}{|c|}{2013} & \multicolumn{2}{|c|}{2018} \\
\hline & UE & $\%$ & UE & $\%$ & UE & $\%$ & UE & $\%$ \\
\hline Agricultura, cría y explotación de animales, aprovechamiento forestal, pesca y caza & 0 & $0 \%$ & 0 & $0 \%$ & 1 & $0 \%$ & 1 & $0 \%$ \\
\hline Minería & 0 & $0 \%$ & 1 & $0 \%$ & 0 & $0 \%$ & 3 & $0 \%$ \\
\hline Energía eléctrica, suministro de agua y de gas natural. & 1 & $0 \%$ & 1 & $0 \%$ & 1 & $0 \%$ & 1 & $0 \%$ \\
\hline Construcción & 0 & $0 \%$ & 0 & $0 \%$ & 2 & $0 \%$ & 1 & $0 \%$ \\
\hline Industrias manufactureras & 98 & $10 \%$ & 155 & $11 \%$ & 130 & $11 \%$ & 175 & $12 \%$ \\
\hline Comercio al por mayor & 30 & $3 \%$ & 32 & $2 \%$ & 30 & $3 \%$ & 45 & $3 \%$ \\
\hline Comercio al por menor & 572 & $58 \%$ & 736 & $53 \%$ & 614 & $51 \%$ & 656 & $45 \%$ \\
\hline Transportes, correos y almacenamiento & 14 & $1 \%$ & 8 & $1 \%$ & 5 & $0 \%$ & 7 & $0 \%$ \\
\hline Información en medios masivos & 3 & $0 \%$ & 4 & $0 \%$ & 2 & $0 \%$ & 3 & $0 \%$ \\
\hline Servicios financieros y de seguros & 0 & $0 \%$ & 5 & $0 \%$ & 9 & $1 \%$ & 9 & $1 \%$ \\
\hline Servicios inmobiliarios y de alquiler de bienes muebles e intangibles & 9 & $1 \%$ & 31 & $2 \%$ & 23 & $2 \%$ & 33 & $2 \%$ \\
\hline Servicios profesionales, científicos y técnicos & 13 & $1 \%$ & 21 & $2 \%$ & 19 & $2 \%$ & 24 & $2 \%$ \\
\hline Servicios de apoyo a los negocios y manejo de residuos & 3 & $0 \%$ & 18 & $1 \%$ & 15 & $1 \%$ & 14 & $1 \%$ \\
\hline Servicios educativos & 11 & $1 \%$ & 11 & $1 \%$ & 13 & $1 \%$ & 16 & $1 \%$ \\
\hline Servicios de salud y de asistencia social & 25 & $3 \%$ & 40 & $3 \%$ & 38 & $3 \%$ & 50 & $3 \%$ \\
\hline Servicios de esparcimiento culturales y deportivos, y otros servicios recreativos & 6 & $1 \%$ & 20 & $1 \%$ & 17 & $1 \%$ & 17 & $1 \%$ \\
\hline Servicios de alojamiento temporal y de preparación de alimentos y bebidas & 91 & $9 \%$ & 101 & $7 \%$ & 110 & $9 \%$ & 187 & $13 \%$ \\
\hline Otros servicios excepto actividades gubernamentales & 111 & $11 \%$ & 193 & $14 \%$ & 170 & $14 \%$ & 220 & $15 \%$ \\
\hline Total & 987 & & 1377 & & 1199 & & 1462 & \\
\hline
\end{tabular}

Fuente: Censos Económicos 2003, 2008, 2013 y 2018 del Instituto Nacional de Estadística y Geografía. 
Cuadro 6. Unidades económicas por sector de actividad del Pueblo Mágico de Viesca, 2003-2018.

\begin{tabular}{|c|c|c|c|c|c|c|c|c|}
\hline \multirow[b]{2}{*}{ Sector económico } & \multicolumn{2}{|c|}{2003} & \multicolumn{2}{|c|}{2008} & \multicolumn{2}{|c|}{2013} & \multicolumn{2}{|c|}{2018} \\
\hline & UE & $\%$ & UE & $\%$ & UE & $\%$ & UE & $\%$ \\
\hline Agricultura, cría y explotación de animales, aprovechamiento forestal, pesca y caza & 0 & $0 \%$ & 12 & $9 \%$ & 6 & $5 \%$ & 20 & $14 \%$ \\
\hline Minería & 0 & $0 \%$ & 0 & $0 \%$ & 0 & $0 \%$ & 1 & $1 \%$ \\
\hline Energía eléctrica, suministro de agua y de gas natural. & 1 & $1 \%$ & 1 & $1 \%$ & 1 & $1 \%$ & 1 & $1 \%$ \\
\hline Construcción & 0 & $0 \%$ & 0 & $0 \%$ & 1 & $1 \%$ & 2 & $1 \%$ \\
\hline Industrias manufactureras & 4 & $4 \%$ & 8 & $6 \%$ & 5 & $4 \%$ & 10 & $7 \%$ \\
\hline Comercio al por mayor & 2 & $2 \%$ & 2 & $2 \%$ & 1 & $1 \%$ & 3 & $2 \%$ \\
\hline Comercio al por menor & 70 & $74 \%$ & 81 & $62 \%$ & 74 & $66 \%$ & 70 & $48 \%$ \\
\hline Transportes, correos y almacenamiento & 8 & $9 \%$ & 0 & $0 \%$ & 0 & $0 \%$ & 0 & $0 \%$ \\
\hline Servicios inmobiliarios y de alquiler de bienes muebles e intangibles & 1 & $1 \%$ & 3 & $2 \%$ & 2 & $2 \%$ & 2 & $1 \%$ \\
\hline Servicios de apoyo a los negocios y manejo de residuos, y servicios de remediación & 1 & $1 \%$ & 2 & $2 \%$ & 1 & $1 \%$ & 0 & $0 \%$ \\
\hline Servicios de salud y de asistencia social & 0 & $0 \%$ & 3 & $2 \%$ & 3 & $3 \%$ & 3 & $2 \%$ \\
\hline Servicios de alojamiento temporal y de preparación de alimentos y bebidas & 2 & $2 \%$ & 7 & $5 \%$ & 13 & $12 \%$ & 26 & $18 \%$ \\
\hline Otros servicios excepto actividades gubernamentales & 5 & $5 \%$ & 11 & $8 \%$ & 5 & $4 \%$ & 7 & $5 \%$ \\
\hline Total & 94 & & 130 & & 112 & & 145 & \\
\hline
\end{tabular}

Fuente: Censos Económicos 2003, 2008, 2013 y 2018 del Instituto Nacional de Estadística y Geografía. 


\section{REFERENCIAS}

Aguirre, L., Tobón G. y Mendoza R. (2018) Dinámica de las regiones de Coahuila: entre las fuerzas de la cuarta revolución industrial, el asalto a sus recursos naturales y la luchas por el espacio. México: Universidad Nacional Autónoma de México y Asociación Mexicana de Ciencias para el Desarrollo Regional A.C.

Equihua, G., Messina, S. y Ramírez J.P. (2015). Los pueblos mágicos: una visión crítica sobre su impacto en el desarrollo sustentable del turismo. Revista Fuente Nueva Época, Año 6, núm. 22, agosto-octubre 2015.

Fernández, A. (2016). Una revisión del programa Pueblos Mágicos. Revista Cultur, año 10, núm. 1, 4-34.

García, D. y Guerrero, H. (2014). El programa Pueblos Mágicos: análisis de los resultados de una consulta local ciudadana. El caso de Cuitzeo, Michoacán, México. Economía y Sociedad, vol. XVII (31), 71-94.

Hofmann, A. (2015). Pueblos mágicos: de la magia al desarrollo local. Buen Gobierno, núm. 19, 106-120.

Instituto Nacional de Estadística y Geografía (2016). Esperanza de vida de los negocios en México. Recuperado de: https://www.inegi.org.mx/temas/evnm/

Instituto Nacional de Estadística y Geografía (2021). Censo de Población y Vivienda 2020. Recuperado de: https://censo2020.mx/

López, L., Valverde, C., Fernández A. y Figueroa M. (2015). Pueblos Mágicos. Una visión interdisciplinaria. México; Universidad Autónoma Metropolitana y Universidad Nacional Autónoma de México.

Organización Mundial de Turismo (2020). Tablero de datos del turismo. Disponible en: https://www.unwto.org/es/unwto-tourism-dashboard

Rosas, M., Almeraya X. y Guajardo G. (2017). Los Comités Pueblos Mágicos y el desarrollo turístico: Tepotzotlán y El Oro, Estado de México. Agricultura, Sociedad y Desarrollo, vol. 14 (1), 105-123.

Secretaria de Turismo (2020). Guía de incorporación y permanencia de los Pueblos Mágico. México: Secretaría de Turismo. Shaadi R., Pulido, J. y Rodríguez I. (2017). El producto turístico en los Pueblos Mágicos de México. Un análisis crítico de sus componentes. Revista de Estudios Regionales, núm. 108, 125-163.

Velarde, M., Maldonado, A. y Maldonado, M. (2009). Pueblos mágicos. Estrategia para el desarrollo turístico sustentable: caso Sinaloa. Teoría y Praxis, núm. 6, 79-93. 